\title{
Erros de administração de antimicrobianos identificados em estudo multicêntrico brasileiro
}

\author{
Tatiane Cristina Marques', Adriano Max Moreira Reis ${ }^{1,2}$, Ana Elisa Bauer de Camargo Silva ${ }^{1,3}$, \\ Fernanda Raphael Escobar Gimenes ${ }^{1,4}$, Simone Perufo Opitz ${ }^{5}$, Thalyta Cardoso Alux Teixeira ${ }^{1}$, \\ Rhanna Emanuela Fontenele Lima', Silvia Helena De Bortoli Cassiani'
}

\begin{abstract}
${ }^{1}$ Departamento de Enfermagem Geral e Especializada, Escola de Enfermagem de Ribeirão Preto, Universidade de São Paulo, ${ }^{2}$ Departamento de Produtos Farmacêuticos, Faculdade de Farmácia, Universidade Federal de Minas Gerais, ${ }^{3}$ Faculdade de Enfermagem, Universidade Federal de Goiás, ${ }^{4}$ Curso de Enfermagem, Universidade Camilo Castelo Branco, ${ }^{5}$ Departamento de Ciências da Saúde e do Desporto, Universidade Federal do Acre
\end{abstract}

*Correspondência:

T. C. Marques

Departamento de Enfermagem Geral e Especializada

Escola de Enfermagem de Ribeirão Preto

Universidade de São Paulo

Av. Bandeirantes, 3900 - Monte Alegre 14040-902 - Ribeirão Preto - SP, Brasil E-mail: tatianecm@hotmail.com
Erros de administração de antimicrobianos são relevantes, pois podem interferir na segurança do paciente e no desenvolvimento de resistência microbiana. O objetivo desse estudo foi identificar os antimicrobianos associados a erros de administração de medicamentos. Estudo multicêntrico, descritivo e exploratório, realizado em unidades de clínica médica de cinco hospitais por meio de técnica observacional, durante 30 dias. Os erros foram classificados em categorias: dose, medicamento não prescrito, via, paciente e horário. A classificação farmacológica dos antimicrobianos foi realizada segundo o Sistema Anatômico Terapêutico Químico (ATC). Os fármacos de intervalo terapêutico estreito foram identificados. A análise estatística descritiva foi realizada no software SPSS 11.5. Foram identificados 1500 erros, sendo 277 (18,5\%) com antimicrobianos. Os tipos de erros foram: de horário $87,7 \%$; de dose $6,9 \%$; de medicamento não autorizado $3,2 \%$, de via $1,5 \%$ e de paciente $0,7 \%$. Foram identificados 36 antimicrobianos e as classes ATC mais freqüentes foram: fluorquinolonas $13,9 \%$, combinações de penicilinas $13,9 \%$, macrolídeos $8,3 \%$ e cefalosporina de terceira geração 5,6\%. Os fármacos de intervalo terapêutico estreito corresponderam a $16,7 \%$ dos antimicrobianos. Os erros com antimicrobianos analisados podem ser fontes de estudo e melhoria no processo de utilização racional de medicamentos e segurança do paciente.
Unitermos

- Medicação/erros de administração

- Agentes antimicrobianos/ erros de administração

- Sistema de medicação hospitalar 


\section{INTRODUÇÃO}

Atualmente, a segurança do paciente é uma das prioridades dos serviços de saúde e compreende um conjunto de iniciativas para instituir sistemas e processos operacionais com o objetivo de evitar, prevenir e reduzir eventos adversos ocorridos a partir da assistência prestada (Manasse Junior, Thompson, 2005). Diante da magnitude do problema dos eventos adversos na assistência à saúde, em especial os erros de medicação, a Organização Mundial de Saúde criou, em 2002, a World Alliance for Patienty Safety. Essa iniciativa visa a incentivar os países a implementarem e monitorarem ações direcionadas à segurança do paciente (Donaldson, Fletcher, 2006).

Os erros de medicação comprometem a segurança do paciente e a qualidade do cuidado. Dentre as conseqüências desses eventos, podem-se destacar: aumento da morbimortalidade relacionada aos medicamentos, prolongamento do tempo de internação e elevação significativa dos custos assistenciais (Bates et al., 1995). Os custos para o sistema americano de saúde, devidos à morbidade e à mortalidade relacionada aos medicamentos, ultrapassam 177 bilhões de dólares por ano desde o ano 2000 (Ernst, Grizzle, 2001; Manasse Junior, Thompson, 2005).

Os fatores que aumentam o potencial para erros de medicação são múltiplos: falta de profissionais de saúde; excesso de trabalho; carga horária pesada; maior número de pacientes exigindo cuidados de alta complexidade e com polifarmacoterapia; crescimento no número, variedade $\mathrm{e}$ potencial de toxicidade dos medicamentos; complexidade tecnológica para o cuidado e o aumento da pressão para reduzir custos e aumentar resultados (Hatcher et al., 2004; Miasso et al., 2006). Profissionais de saúde, mesmo altamente capacitados e experientes, quando expostos a ambientes de trabalho com estas características, podem cometer erros devidos a tais fatores sistêmicos.

O sistema de utilização de medicamentos engloba vários processos interligados e interdependentes, envolvendo multiplicidade de planejamento e implementação de ações pela equipe de saúde e gerando um contexto entrelaçado de situações que podem ser facilitadoras para ocorrência de um erro (Ackroyd-Stolarz, Hartnell, MacKinnon, 2005; Dang et al., 2007). A incidência de erro de medicação é diferenciada ao longo de toda a cadeia de utilização. Estudo realizado por Koop et al. (2006) identificou que de um total de 132 erros de medicação, $36 \%$ ocorreram na fase de prescrição, 32\% na administração, $28 \%$ na dispensação e $4 \%$ na transcrição dos medicamentos prescritos.

Recentemente, os erros de medicação, assim como o uso inadequado de medicamentos, passaram a ser considerados um problema de saúde pública em função do impacto assistencial e da elevada incidência nos serviços de saúde (Manasse Junior, Thompson, 2005). A dimensão do problema fica bem caracterizada por estudos epidemiológicos divulgados pelo Institute of Medicine, que estimam que cada paciente internado em hospitais norte-americanos esteja sujeito a um erro de medicação por dia, e que, anualmente, ocorrem nesses serviços de saúde, aproximadamente, 400.000 eventos adversos evitáveis relacionados a medicamentos (Bates, 2007).

Estudos que se propõem a analisar os tipos de erros de medicação e suas causas, assim como as classes farmacológicas envolvidas, são relevantes para evidenciar os problemas existentes no processo de medicação. Investigações realizadas para analisar erros de medicação segundo a classe terapêutica, identificaram que a freqüência desse evento com antimicrobianos varia de $4,9 \%$ a $39 \%$ (Rissato, 2005). Esses resultados são preocupantes, pois os antimicrobianos representam uma das classes mais prescritas em hospitais, sendo responsáveis por uma parcela elevada das despesas com medicamentos. É também crescente a preocupação com o uso inadequado, considerando-se que esse constitui o principal fator associado ao aparecimento de resistência microbiana (Murthy, 2001; Muto et al., 2003; Levy, Marshall, 2004).

A identificação dos erros de medicação com antimicrobianos pode auxiliar no desenvolvimento de novas práticas que garantam o uso adequado e racional dos medicamentos, aumentando, assim, a segurança do paciente. Dessa maneira, os objetivos desse estudo foram identificar e analisar os erros de medicação com antimicrobianos durante o processo de administração de medicamentos em cinco hospitais brasileiros, e determinar as características farmacológicas dos antimicrobianos associados a erros.

\section{MATERIAL E MÉTODOS}

Trata-se de um estudo multicêntrico, com delineamento exploratório, que visa a obter informações detalhadas da variável erro de medicação em hospitais brasileiros, buscando determinar a frequência e os fatores associados a ocorrência do evento. Considerou-se como erro qualquer discrepância entre o que estava prescrito e o que foi administrado pela enfermagem ao paciente. Nesta publicação, são apresentados os dados referentes aos erros na administração de medicamentos antimicrobianos.

A população em estudo foi composta pelas situações em que houve erro de medicação, ou seja, 1500 erros identificados em um total de 4958 observações. A unidade de análise foi a administração do medicamento.

A pesquisa foi desenvolvida em unidades de clínica médica de cinco hospitais situados nas regiões Norte, Nor- 
deste, Sudeste e Centro-Oeste do Brasil. As instituições investigadas foram selecionadas, por possuírem vínculo com universidades públicas estaduais ou federais, fazerem parte da Rede de Hospitais Sentinela da Agência Nacional de Vigilância Sanitária (Anvisa) e serem campo de estágio para as instituições formadoras de profissionais de saúde do País. A unidade de clínica médica foi selecionada, por possuir leitos destinados a pacientes portadores de doenças crônico-degenerativas que, usualmente, utilizam um número elevado de medicamentos durante um longo período, englobando diversas classes terapêuticas.

A investigação foi realizada após a aprovação dos Comitês de Ética em Pesquisa e após autorização da direção dos hospitais investigados. Todos os participantes foram orientados sobre o estudo, aceitaram, por escrito, serem observados e assinaram o termo de consentimento livre e esclarecido, conforme a Resolução 196/96 do Conselho Nacional de Saúde (Brasil, 1996).

Para coleta dos dados, foram realizadas observações não participantes e diretas das atividades dos profissionais de enfermagem responsáveis pela administração de medicamentos. Para tanto, quinze auxiliares de pesquisa receberam treinamento de vinte horas e durante 30 dias realizaram as observações seguindo um roteiro estruturado validado por especialistas envolvidos com a temática. Após as observações da administração dos medicamentos, os observadores confrontaram as informações obtidas com as prescrições, a fim de detectar se ocorreu algum erro de medicação. Para cada hospital, foram designados três auxiliares de pesquisa e a coleta foi realizada no mesmo período em todos os centros investigados.

Os erros de medicação foram classificados segundo a tipologia da American Society of Health System Pharmacists: erro de dose, erro de medicamento não prescrito, erro de via, erro de paciente e erro de horário (ASHP, 1993). Foi conside- rado erro de horário a situação em que o medicamento foi administrado em um período superior a 60 minutos de antecedência ou de atraso em relação ao horário de administração definido na prescrição ou no plano de administração elaborado pelo enfermeiro ou técnico de enfermagem.

A classificação farmacológica dos medicamentos antimicrobianos identificados nos erros foi realizada segundo o sistema anatômico terapêutico químico (ATC) do WHO Collaborating Center for Drug Statistics Methodology (WHO, 2007), e empregado pelo Centro Colaborador para o Monitoramento Internacional de Medicamentos. Os fármacos de intervalo terapêutico estreito foram identificados em fonte terciária de referência, o Drugdex (Klasco, 2007).

Os dados coletados foram transferidos para um banco de dados, elaborado no programa Epi data 3.1, com dupla digitação, validação e checagem da consistência das informações. A análise estatística foi realizada empregando o software SPSS 11.5 e consistiu de análise descritiva univariada com determinação das freqüências absolutas e relativas.

\section{RESULTADOS E DISCUSSÃO}

Os resultados permitiram, em uma primeira abordagem, identificar e analisar os erros de medicação com antimicrobianos durante o processo da administração de medicamentos. Foram realizadas 4958 observações da administração dos medicamentos, nas quais foram identificados 1500 (30,24\%) erros de medicação. Desse total, 277 $(18,5 \%)$ erros envolveram medicamentos antimicrobianos e essa incidência é compatível com outros estudos publicados na literatura internacional (Benjamin, 2003; Wolf, Hicks, Serembus, 2006; Tang et al., 2007).

Os erros com medicamentos antimicrobianos foram categorizados e estratificados por forma farmacêutica, conforme apresentado na Tabela I.

TABELA I - Distribuição dos tipos de erros de medicação com antimicrobianos estratificados por forma farmacêutica, identificados no processo de administração de medicamentos na unidade de clínica médica em cinco hospitais sentinela, Brasil, 2006

\begin{tabular}{|c|c|c|c|c|c|c|}
\hline \multirow[t]{2}{*}{ Tipo de erro } & \multicolumn{4}{|c|}{ Formas Farmacêuticas dos Medicamentos } & \multicolumn{2}{|c|}{ Total } \\
\hline & $\begin{array}{c}\text { Uso } \\
\text { parenteral (n) }\end{array}$ & $\begin{array}{l}\text { Uso oral } \\
\text { sólida (n) }\end{array}$ & $\begin{array}{l}\text { Uso oral } \\
\text { Líquida (n) }\end{array}$ & $\begin{array}{c}\text { Uso } \\
\text { Oftálmico (n) }\end{array}$ & $\mathrm{N}$ & $\%$ \\
\hline Erro de horário & 230 & 13 & & & 243 & 87,7 \\
\hline Erro de dose & 17 & 2 & & & 19 & 6,9 \\
\hline $\begin{array}{l}\text { Erro de medicamento } \\
\text { não autorizado }\end{array}$ & 3 & 1 & 4 & 1 & 9 & 3,2 \\
\hline Erro de via & 1 & 2 & 1 & & 4 & 1,5 \\
\hline Erro de paciente & 2 & & & & 2 & 0,7 \\
\hline Total & $253(91,3 \%)$ & $18(6,5 \%)$ & $5(1,8 \%)$ & $1(0,4 \%)$ & 277 & 100,0 \\
\hline
\end{tabular}


A categoria de erro mais freqüente foi a de horário, envolvendo $243(87,7 \%)$ medicamentos antimicrobianos administrados, sendo que, desses, 140 (57,6\%) foram administrados com antecedência em relação ao horário planejado.

A alta incidência de erros de horário possivelmente tem como principais determinantes fatores internos ao processo de administração, como o planejamento de horário pela equipe de enfermagem, que concentra um número elevado de medicamentos em determinados períodos, geralmente o da manhã, fazendo com que aqueles que requerem pontualidade na administração, como antimicrobianos, não tenham seu horário cumprido, devido à alta demanda de serviço na unidade (Manias, Aitken, Dunning, 2005; Miasso et al., 2006). Outro fator que pode gerar erro de horário é o funcionamento inadequado do sistema de distribuição de medicamentos da farmácia hospitalar, que leva a atrasos na entrega dos medicamentos e, conseqüentemente, na sua administração (Carvalho, Cassiani, 2000).

O desconhecimento das especificidades dos antimicrobianos a serem administrados, assim como da necessidade de se cumprirem os intervalos de tempo entre as doses pode ser mais um fator que contribui para a ocorrência deste tipo de erro. A divulgação de informações sobre antimicrobianos e a capacitação da equipe de enfermagem podem ser estratégias de prevenção de erros (Hoefel, Lautert, 2006a).

Para entender o impacto dos erros com antimicrobianos na segurança do paciente, é importante destacar que a sua utilização envolve a aplicação de dois componentes farmacológicos distintos, o farmacocinético e o farmacodinâmico (Mouton et al., 2002; Castro, 2005).

O componente farmacocinético descreve os processos de absorção, distribuição e eliminação dos antimicrobianos, que determinam as concentrações sanguíneas e tissulares para uma determinada dose. Os parâmetros farmacocinéticos mais utilizados em terapia antimicrobiana são: a área abaixo da curva da concentração sanguínea versus o tempo após a administração do medicamento, a concentração sanguínea máxima e a meia vida de eliminação, que está diretamente relacionada ao intervalo de administração do antimicrobiano (Mouton et al., 2002; Castro, 2005).

O componente farmacodinâmico relaciona as concentrações sanguíneas com a atividade antimicrobiana avaliada in vitro assim como a toxicidade sobre o hospedeiro. Os parâmetros farmacodinâmicos, que são freqüentemente empregados para quantificar a atividade antimicrobiana para determinado patógeno, são as concentrações inibitória mínima e a bactericida mínima. Esses parâmetros são utilizados como valores críticos para interpretação da relação entre os parâmetros farmacocinéticos que determinam a eficácia do antimicrobiano e a sua potência. São empregados também na padronização de doses e de intervalos de administração (Castro, 2005).

A Tabela I também mostra que os erros de dose foram a segunda categoria mais freqüente, envolvendo dezenove $(6,9 \%)$ medicamentos. Estes erros podem estar associados à falhas no cálculo matemático durante o preparo do medicamento (Fry, Dacey, 2007). Na casuística desse estudo, os erros de dose são preocupantes, considerando-se que, em unidades de clínica médica, é comum a internação de pacientes geriátricos, que apresentam diminuição da função hepática e renal, assim como a diminuição do metabolismo e da eliminação dos medicamentos (Merle et al., 2005; Hodgkinson et al., 2006).

A grande preocupação dos profissionais de saúde se dá com uma administração de doses superiores à prescrita, o que pode causar graves eventos adversos ao paciente, entretanto, deve haver preocupação também com a administração de doses inferiores de antimicrobianos. Não pode ser ignorado que existem riscos iminentes e que a recorrência de administração de doses superiores ou inferiores ou com intervalos inadequados em um mesmo paciente podem levar ao insucesso terapêutico (Hoefel, Lautert, 2006a).

Apesar de não existirem pesquisas que demonstrem que os erros de administração influenciam na efetividade terapêutica e na resistência bacteriana, a equipe de saúde deve estar atenta para a sua prevenção, principalmente quando os medicamentos envolvidos são antimicrobianos, considerando-se que a resistência bacteriana está associada ao uso inadequado desses fármacos. De acordo com Hoefel \& Lautert (2006a), a equipe de enfermagem é responsável por assegurar uma monitorização rigorosa da administração de antimicrobianos com o controle dos horários, diluições e intervalos entre as doses, evitando a seleção de microorganismos resistentes devido a níveis plasmáticos inadequados.

A interferência dos erros de administração de antimicrobianos sobre os componentes farmacocinético e farmacodinâmico e a inter-relação com a resistência microbiana foi pontuada por Hoefel \& Lautert (2006b). As autoras alertam que, independentemente do número de vezes em que doses incompletas de antimicrobianos são administradas, o que deve ser considerado é o fato de que o erro de administração pode levar a concentrações plasmáticas inferiores à concentração inibitória mínima, exercendo pressão seletiva sobre o microrganismo. Essa pressão seletiva é um dos fatores de risco para o aparecimento da resistência microbiana.

Neste contexto, fica evidente que os erros de dose e de horário podem comprometer a resposta terapêutica do antimicrobiano. A administração errada do antimicrobiano 
TABELA II - Distribuição dos principais antimicrobianos associados com erros de medicação identificados no processo de administração de medicamentos na unidade de clínica médica em cinco hospitais sentinela, Brasil, 2006

\begin{tabular}{lcccccc}
\hline Antimicrobianos & $\begin{array}{c}\text { Erro de } \\
\text { Paciente }\end{array}$ & $\begin{array}{c}\text { Erro de } \\
\text { Via }\end{array}$ & $\begin{array}{c}\text { Erro Medicamento } \\
\text { não autorizado }\end{array}$ & $\begin{array}{c}\text { Erro de } \\
\text { Dose }\end{array}$ & $\begin{array}{c}\text { Erro de } \\
\text { Horário }\end{array}$ & Total \\
\hline Ceftriaxona - Frasco ampola & & 1 & & 1 & 34 & 36 \\
Vancomicina - Frasco ampola & 1 & & & 3 & 23 & 27 \\
Clindamicina - Ampola & & & & 3 & 21 & 24 \\
Oxacilina - Frasco ampola & & & & & 23 & 23 \\
Cefepime - Frasco Ampola & & 1 & 1 & 18 & 20 \\
Imipenem + Cilastatina - Frasco ampola & & & & 3 & 14 & 17 \\
Metronidazol - Frasco ampola & & & & 1 & 15 & 16 \\
Ciprofloxacina - Frasco ampola & 1 & & & & 14 & 14 \\
Ceftazidma - Frasco ampola & & 1 & & 1 & 9 & 11 \\
Ciprofloxacina - comprimido & & 2 & 8 & 6 & 62 & 78 \\
Outros & 2 & 4 & 9 & 19 & 243 & 277 \\
Total & & & & & & \\
\hline
\end{tabular}

pode impedir que ele alcance o local da infecção, mantenha concentrações suficientes no foco da infecção para exercer sua ação, e permaneça no local tempo suficiente para inibir a multiplicação ou matar os microorganismos.

Ao analisar as formas farmacêuticas dos medicamentos, as de uso parenteral foram as mais freqüentemente envolvidas nos erros com antimicrobianos estando presentes em $253(91,3 \%)$ erros. Vale destacar que $230(94,65 \%)$ erros de horário e dezessete $(89,5 \%)$ erros de dose envolveram antimicrobianos na forma para uso parenteral.

A administração de medicamentos antimicrobianos pela via parenteral corresponde a uma proporção significativa nos hospitais. Erros relacionados com a administração parenteral, principalmente os que envolvem a via endovenosa, podem apresentar ainda maior gravidade, considerando-se que a dose é administrada diretamente na corrente sanguínea, levando a um efeito rápido, sendo necessário monitorizar o paciente para a ocorrência de reações adversas. Além disso, os níveis plasmáticos do medicamento podem elevar-se e causar toxicidade, quando administrados em "bolus" ou no caso dos tratamentos prolongados (Santell, Cousins, 2005).

Com o intuito de proporcionar maior conforto ao paciente, menor risco de adquirir uma infecção hospitalar pelo uso do cateter e redução de custos, devem ser desenvolvidas políticas institucionais que estimulem a troca da terapia parenteral, principalmente a intravenosa, pela via oral, quando possível (Gunten et al., 2003).

Em uma segunda abordagem, este estudo analisou os principais antimicrobianos envolvidos nos 277 erros ocorridos no processo de administração de medicamentos. $\mathrm{Na}$ Tabela II, eles serão apresentados segundo as categorias de erros de medicação e, na Tabela III, conforme as classes anatômica terapêutica-química. Vale salientar que, na administração de um mesmo medicamento, foi identificado mais de um erro.

TABELA III - Distribuição quanto à classe ATC dos antimicrobianos envolvidos nos erros de medicação no processo de administração de medicamentos em unidades de clínica médica em cinco hospitais sentinela segundo a Classificação ATC - Nível IV, Brasil, 2006

\begin{tabular}{lcc}
\hline Classsificação ATC - Nível IV & N & $\%$ \\
\hline Fluorquinolonas & 5 & 13,9 \\
Associação de penicilinas com & 5 & 13,9 \\
inibidores de beta lactamase & & \\
Macrolídeos & 3 & 8,3 \\
Aminoglicosídeos & 2 & 5,6 \\
Antifúngico derivado triazólico & 2 & 5,6 \\
Carbapenêmico & 2 & 5,6 \\
Cefalosporina de primeira geração & 2 & 5,6 \\
Cefalosporina de terceira geração & 2 & 5,6 \\
Lincosamidas & 2 & 5,6 \\
Penicilinas de amplo espectro & 2 & 5,6 \\
Penicilinas sensíveis a beta lactamase & 2 & 5,6 \\
Antibacterianos derivados imidazólicos & 2 & 5,6 \\
Antifúngico derivado imidazólico & 1 & 2,8 \\
Antibiótico & 1 & 2,8 \\
Cefalosporina de quarta geração & 1 & 2,8 \\
Glicopeptideos & 1 & 2,8 \\
Outros fármacos para & 1 & 2,8 \\
tratamento da tuberculose & & \\
Total & 36 & 100,0 \\
\hline
\end{tabular}


Entre os antimicrobianos associados com erros estão as cefalosporinas de terceira geração e as penicilinas de amplo espectro, grupos que têm contribuído para o aumento da resistência de bactérias produtoras de beta lactamase extendida. Atualmente, esses microrganismos também representam uma grande preocupação referente à resistência bacteriana (Hoefel, Lautert, 2006b).

A ceftriaxona, cefalosporina de terceira geração, foi o medicamento envolvido no maior número de erros no presente estudo. A elaboração conjunta pela equipe de enfermagem e de farmácia de um manual de utilização de medicamentos abordando aspectos como preparo, esquemas de administração, interações, incompatibilidades, reações adversas é uma iniciativa que contribui para a segurança da utilização de medicamentos (Bucknall, 2007).

A Tabela II também aponta que os erros detectados durante a administração de vancomicina, fármaco de intervalo terapêutico estreito, correponderam a segunda posição em frequência absoluta. Ressalta-se que houve erros de dosagem e que a superdosagem de vancomicina pode gerar nefrotoxicidade. Outra consequência da administração inadequada é a síndrome do homem do pescoço vermelho, que pode ocorrer devido a erros de diluição e no tempo de administração, trazendo danos ao paciente (Klasco, 2007).

Entretanto, a maior preocupação é com a resistência dos microrganismos à vancomicina. A redução da sensibilidade do Staphylococcus aureus à vancomicina representa uma grande preocupação, por se tratar de uma das últimas opções terapêuticas a esse microrganismo. Outra questão alvo de maior ou igual preocupação é a resistência de Enterococcus à vancomicina (Muto et al., 2003). O surgimento de resistência pode ser favorecido por erros de horário e de dose.

Neste estudo também foi identificado que seis $(16,7 \%)$ medicamentos antimicrobianos associados com erro possuíam intervalo terapêutico estreito. Vale destacar que $47,4 \%$ dos erros de dose e $21,8 \%$ dos erros de horário envolveram antimicrobianos de intervalo terapêutico estreito, sendo vancomicina, amicacina e clindamicina os principais fármacos associados. Diante de medicamentos com essa característica, é possivel inferir sobre a possibilidade de danos aos pacientes devidos à superdosagem e de impacto sobre a resistência devido aos níveis plasmáticos reduzidos.

Deve ser ressaltado, também, que os medicamentos de intervalo terapêutico estreito necessitam de monitorização terapêutica, a fim de determinar a dose que produza o máximo de efetividade aliada ao mínimo de efeitos tóxicos. O conhecimento da equipe de enfermagem sobre medicamentos de intervalo terapêutico estreito é importante para garantir tanto a administração correta do medicamentos como o preparo correto do paciente, assim como para a decisão e programação multidisciplinar de coleta das amostras sanguíneas, quando a monitorização laboratorial for necessária.

A magnitude do problema dos erros com antimicrobianos em nosso meio foi primeiro demonstrado em uma pesquisa enfocando iatrogenia com antibióticos em terapia intensiva que detectou uma discordância de $38,6 \%$ entre o número de doses prescritas e o número de doses administradas e redução de $85 \%$ no número de doses administradas (Manenti et al., 1998). Estudo mais recente analisou erros de administração com um antimicrobiano específico, o cefepime, demonstrando que o problema continua ocorrendo em serviços de saúde (Hoefel, Lautert, 2006b).

Os 277 erros envolveram 36 medicamentos antimicrobianos, sendo 32 antibacterianos e quatro antifúngicos. Na Tabela III pode ser verificado que as fluorquinolonas $(13,9 \%)$, as associações de penicilinas com inibidores de beta lactamase $(13,9 \%)$ e os macrolídeos $(8,3 \%)$ foram as classes mais freqüentes.

A resistência às quinolonas, classe terapêutica mais freqüente neste estudo, é um problema emergente, devido ao crescente número de cepas hospitalares de Enterobacteriaceae e Pseudomonas aeruginosa resistentes às quinolonas. Investigações buscando elucidar os fatores desencadeantes dessa resistência verificaram que a redução das concentrações plasmáticas devida à diminuição da absorção por interação com cátions diou trivalentes pode explicá-la (Quain et al., 2005, Barton et al., 2005). Os erros de horário podem levar à ocorrência de interações que comprometem a absorção, pois aumentam a probabilidade de co-administração de medicamentos.

A incidência das infecções fúngicas teve um aumento significativo nos últimos anos. Paralelamente, pode ser observada uma gravidade maior dessas infecções, principalmente nos pacientes hospitalizados (Lagrou et al., 2007). Sendo assim, a identificação de quatro antifúngicos associados com erros de administração desperta preocupação, considerando-se a situação clínica dos pacientes para os quais é prescrita essa terapêutica.

Assim como o uso irracional, os erros de medicação com antimicrobianos têm conseqüências individuais e coletivas, porque, além de afetarem o indivíduo que faz uso do medicamento, afetam de maneira significativa a microbiota do ambiente hospitalar. Essas conseqüências abrangem desde a elevação dos gastos com medicamentos, inefetividade terapêutica, aumento da hospitalização devido a eventos adversos, recrudescimento das infecções até o mais grave, o aumento da resistência microbiana.

É consenso que a prevenção da resistência microbiana envolve ações multidisciplinares centradas principalmente no uso racional de antimicrobianos e na otimização das ações de controle de infecções (Murthy, 2001; Muto et al., 2003). As publicações internacionais apresentam as atividades do 
farmacêutico e do médico no controle da resistência microbiana, porém, apesar de ressaltarem a participação do enfermeiro no grupo multidisciplinar, não explicitam claramente quais seriam suas ações no controle do uso de antimicrobianos. Diante dessa problemática, Bisset (2006) apresentou um conjunto de recomendações para controle e redução do risco de resistência microbiana e destacou que a equipe de enfermagem deve conhecer a política institucional de antimicrobianos e protocolos de utilização para assegurar que a terapêutica antimicrobiana seja realizada segundo as melhores práticas clínicas.

No sentido de delinear ações de prevenção de eventos adversos com antimicrobianos, é importante considerar que os erros de administração, assim como os de medicação em geral, apresentam múltiplos determinantes. Nessa perspectiva, a identificação dos fatores determinantes dos erros, por meio de ferramentas da qualidade, como a análise de causa raiz, bem como a implementação de medidas preventivas e alterações de processos, são essenciais para o aprimoramento da assistência e para segurança do paciente (Ackroyd-Stolarz, Hartnell, MacKinnon, 2005; Manasse Junior, Thompson, 2005).

Entre as medidas preventivas efetivas podem ser citadas: dupla checagem das diluições e cálculos da dosagem dos medicamentos, atenção aos erros de comunicação advindos da prescrição médica, cuidado com as informações verbais, identificação segura do paciente bem como a orientação sobre sua farmacoterapia (Manasse Junior, Thompson, 2005; Bates, 2007). Outra estratégia recomendada é a participação do farmacêutico clínico na unidade de internação como um elemento-chave dentro da equipe de saúde para realizar o seguimento da farmacoterapia e contribuir para o uso racional de medicamentos (Kucukarslan et al., 2003; Scheneider, 2007).

O conhecimento da equipe de saúde em farmacoterapia contribui significativamente para a prevenção de erros de medicação (Ackroyd-Stolarz, Hartnell, MacKinnon, 2005). Dessa forma, érecomendável que haja investimentos em educação continuada, cursos de reciclagem e treinamentos periódicos, incentivo à realização de investigações científicas sobre o sistema de utilização de medicamentos, inclusive em relação aos erros de medicação, com a finalidade de identificar suas causas e propor melhorias.

\section{CONCLUSÕES}

Os estudos multicêntricos que abordam a temática dos erros de medicação, usando técnica de observação não participante, são estratégias adequadas para conhecer a utilização de medicamentos em hospitais e contribuir para a implementação de medidas preventivas. Investigações devem ser realizadas para melhorar a prática e aumentar a segurança do paciente na utilização dos antimicrobianos e garantir seu uso racional evitando os erros de medicação.

Ações para promover o uso racional de antimicrobianos envolvendo todas as fases do processo de utilização devem ser desenvolvidas pelas comissões de farmácia e terapêutica e de controle de infecção hospitalar, visando a conter a expansão da resistência microbiana nos serviços de saúde. Os profissionais de saúde presentes nessas comissões devem, ao participar do planejamento das ações preventivas e educativas, incorporar aspectos que explicitem claramente suas atividades e funções na prevenção da resistência microbiana e no uso racional de antimicrobianos. Paralelamente, a equipe de saúde deve ser conscientizada da importância do uso seguro de antimicrobianos para o controle da resistência.

Para garantir a segurança do paciente, é essencial conscientizar os profissionais de que uma resposta terapêutica adequada e sem danos ao paciente é responsabilidade de toda a equipe de saúde. A abordagem dos erros de medicação de forma sistêmica é essencial na implantação e melhoria das práticas assistenciais visando maior segurança no cuidado em saúde.

Os dados apresentados demonstram a importância de medidas de prevenção com abordagens multidisciplinar e interdisciplinar e promoção de uma cultura de segurança com enfoque educativo e não punitivo. O erro de medicação é evitável e deve ser analisado e revertido em educação e melhoria do sistema, promovendo o fortalecimento do processo de administração de medicamentos tornando-o mais seguro.

\section{ABSTRACT}

\section{Antimicrobial drug administration errors identified in Brazilian multicentric study}

Medication administration errors (MAE) are the most frequent kind of medication errors. Errors with antimicrobial drugs $(A D)$ are relevant because they may interfere in patient safety and in the development of microbial resistance. The aim of this study is to analyze the AD errors detected in a Brazilian multicentric study of MAE. It was a descriptive and exploratory study carried out in clinical units in five Brazilian teaching hospitals. The hospitals were investigated during 30 days. MAE were detected by observation technique. MAE were classified in categories: wrong route(WR), wrong patient(WP), wrong dose(WD) wrong time (WT) and unordered drug (UD). AD with MAE were classified by Anatomical-Therapeutical-Chemical Classification System. $A D$ with narrow therapeutic index (NTI) were identified. A descriptive statistical analysis was performed using SPSS 
version 11.5 software. A total of 1500 errors were observed, 277 (18.5\%) of them were errors with $A D$. The types of $A D$ error were: WT 87.7\%, WD 6.9\%, WR 1.5\%, UD 3.2\% and WP $0.7 \%$. The number of AD found was 36. The mostly ATC class were fluoroquinolones $13.9 \%$, combinations of penicillin $13.9 \%$, macrolides $8.3 \%$ and third-generation cephalosporins $5.6 \%$. The parenteral drug dosage form was associated with $55.6 \%$ of AD. $16.7 \%$ of AD were NTI. $47.4 \%$ of WD and $21.8 \%$ WT were with NTI drugs. This study shows that these errors should be considered potential areas for improvement in the medication process and patient safety plus there is requirement to develop rational drug use of $A D$.

UNITERMS: Medication/administration errors. Antimicrobial drugs/administration errors. Medication systems hospital.

\section{AGRADECIMENTOS}

Aos coordenadores locais do projeto multicêntrico Flávio Trevisan Fakih e Maria Ludermiler Sabóia Mota. Pesquisa patrocinada pela FAPESP E CNPq.

\section{REFERÊNCIAS BIBLIOGRÁFICAS}

ACKROYD-STOLARZ， S.; HARTNELL, N.; MACKINNON, N.J. Approaches to improving the safety of the medication use system. Healthcare Q., v.8, p.59-64, 2005.

\section{AMERICAN ASSOCIATION OF HOSPITAL} PHARMACISTS. ASHP. Guideline on preventing medication errors in hospitals. Am. J. Hosp. Pharm., v.50, p.305-314, 1993.

BARTON, T.D.; FISHMAN, N.O.; WEINER, M.G.; LAROSA, L.A.; LAUTENBACH, E. High rate of coadministration of di-or-tri-valent cation - containing compounds with oral fluoroquinolones: risk factors and potential implications. Infect. Control and Hosp. Epidemiol., v.26, p.93-99, 2005.

BATES, D.W. Preventing medication errors: a summary. Am. J. Health Syst. Pharm., v.64, p.S3-S9, 2007.

BATES, D.W.; CULLEN, D.J.; LAIRD, N.; PETERSEN, L.A.; SMALL, S.D.; SERVI, D. LAFFEL, G.; SWEITZER, B.J.; SHEA, B.F.; HALLISEY, R.; VLIET, V.M.; NEMESKAL, R.; LEAPE, L. Incidence of adverse drug events and potential adverse drug events: implications for prevention. JAMA, v.274, p.29-34, 1995.
BENJAMIN, D.M. Reducing medication errors and increasing patient safety: case studies in clinical pharmacology. J. Clin. Pharm., v.43, p.768-783, 2003.

BISSETT, L. Reducing the risk of acquiring antimicrobialresistant bacteria. Br. J. Nurs., v.15, n.2, p.68-71, 2006.

BRASIL. Ministério da Saúde. Conselho Nacional de Saúde. Resolução n.196, de 10 de outubro de 1996. Aprova as Diretrizes e Normas Regulamentadoras de Pesquisas com seres humanos. Disponível em: <http:// conselho.saude.gov.br/docs/Resolucoes/Reso196.doc $>$. Acesso em: 30 mar. 2005.

BUCKNALL, T.K. Implementing guidelines to improve medication safety for hospitalized patients: experiences from western health, Australia. Worldviews Evid. Based Nurs., v.4, n.1, p.51-53, 2007.

CARVALHO, V.T.; CASSIANI, S.H.B. Erros na medicação: análise das situações relatadas pelos profissionais de enfermagem. Medicina, v.33, p.322-330, 2000.

CASTRO, M.S. Princípios farmacocinéticos e farmacodinâmicos em antibioticoterapia. In: OLIVEIRA, A.C. Infecções hospitalares epidemiologia prevenção e controle. Rio de Janeiro: Medsi, 2005. p.357-367.

DANG, D.; ROBERT FEROLI, E.; GILL, C.; PAINE, L.; SHERMOCK, K.; SUFLITA, J.; WALRATH, J.M. Quest for the ideal: a redesign of the medication use system. J. Nurs. Care Qual., v.22, n.1, p.11-17, 2007.

DONALDSON, L.J.; FLETCHER, M.J. The WHO world alliance for patient safety: towards the of living less dangerously. Med. J. Aust., v.10, p.S69-S72, 2006.

ERNST, F.R.; GRIZZLE, A.J. Drug-related morbity and mortality: updating the cost-of-ilness model. J. Am. Pharm. Assoc., v.43, n.2, p.192-199, 2001.

FRY, M.M.; DACEY, M. Factors contributing to incidents in medicine administration. Part 1 Br. J. Nurs., v.16, n.9, p.556-559, 2007.

GUNTEN, V.V.; AMOS, V.; SIDLER, A.L.; BENEY, J.; TROILLET, N.; REYMOND, J.P. Hospital pharmacists' reinforcement of guidelines for switching from parenteral to oral antibiotics: a pilot study. Pharm. World Sci., v.25, n.2, p.52-55, 2003. 
HATCHER, I.; SULLIVAN, M.; HUTCHINSON, J.; THURMAN, S.; GAFFNEY, F.A. An intravenous medication safety system preventing high-risk medication errors at the point of care. J. Nurs. Adm., v.34, n.10, p.437-439, 2004.

HODGKINSON, B.; KOCH, G.S.; NAY, R.; NICHOLS, K. Strategies to reduce medications errors with reference to older adults. Int. J. Evid. Based Healthc., v.4, p.2-41, 2006.

HOEFEL, H.H.K.; LAUTERT, L. Errors commited by nursing technicians and assistants in administering antibiotics. Am. J. Infect. Control., v.34, p.437-442, $2006 b$.

HOEFEL, H.H.K.; LAUTERT, L. Administração endovenosa de antibióticos e resistência bacteriana: responsabilidade da enfermagem. Rev. Eletrônica Enferm., v.8, n.3, p.441-449, 2006a. Disponível em: $<$ http://www.fen.ufg.br/revista/revista $8 \_3 /$ v8n3a15.htm.> Acesso em: 25 jul. 2007.

KLASCO, R.K. (ED): DRUGDEX® System. Thomson MICROMEDEX, Greenwood Village, Colorado, USA. 2007. Disponível em: <http:// www.periodicos.capes.gov.br>Acesso em: 25 jul. 2007.

KOOP, B.J.; ERSTAD, B.L.; ALLEN, M.E.; THEODOROU, A.A.; PRIESTLEY, G. Medication errors and adverse drug events in an intensive care unit: direct observation approach for detection. Crit. Care Med., v.34, n.2, p.415-425, 2006.

KUCUKARSLAN, S.N.; PETER, M.; MLYNARECK, M.; NAFZIGER, D.A. Pharmacists on rounding teams reduce preventable adverse drug events in hospital general medicine units. Arch. Intern. Med., v.163, p.2014-2018, 2003.

LAGROU, K.; VERHAEGEN, J.; PEETERMANS, W.E.; DE RIJDT, T.; MAERTENS, J.; VAN WIJNGAERDEN, E. Fungemia at a tertiary care hospital: incidence, therapy, and distribution and antifungal susceptibility of causative species. Eur. J. Clin. Microbiol. Infect. Dis., v.26, p.541-547, 2007.

LEVY, S.B.; MARSHALL, B. Antibacterial resistance worldwide: causes, challenges and responses. Nat. Med., v.10, n.12, p.S122-129, 2004.
MANASSE JUNIOR, H.R.; THOMPSON, K.K. Medication safety as problem in public policy. In: .Medication safety: a guide for health care facilities. Bethesda: ASHP, 2005. p.1-15.

MANENTI, S.; CHAVES, A.B.; LEOPOLDINO, R.S.; PADILHA, K.G. Ocorrências adversas com medicação em unidades de terapia intensiva: análise da administração de soluções hidroeletrolíticas e antibióticos. Rev. Esc. Enferm. USP, v.32, n.4, p.369-376, 1998.

MANIAS, E.; AITKEN, R.; DUNNING, T. How graduate nurses use protocols to manage patients' medications. $J$. Clin. Nurs., v.14, p.935-944, 2005.

MERLE, L.; LAROCHE, M.L.; DANTONE, T.; CHAMES, J.P. Prediciting and preventing adverse drug reactions in very old. Drug Aging, v.22, n.5, p.375-392, 2005.

MIASSO, A.I.; GROU, C.R.; CASSIANI, S.H.B.; SILVA, A.E.B.C.; FAKIH, F.T. Erros de medicação: tipos, fatores causais e providências tomadas em quatro hospitais brasileiros. Rev. Esc. Enferm. USP, v.40, n.4, p.524-532, 2006.

MOUTON, J.W.; DUDLEY, M.N.; CARS, O.; DERENDORF, H.; DRUSANO, G.L. Standardization of pharmacokinetic/pharmacodynamic (PK/PD) terminology for anti-infective drugs. Int. J. Antimicrob. Agents, v.19, n.4, p.355-358, 2002.

MURTHY, R. Implementatation of strategies to control antimicrobial resistance. Chest, v.119, p.405S-411S, 2001.

MUTO, C.A.; JERNIGAN, J.A.; OSTROWSKY, B.R.; RICHET, H.E.; JARVIS, W.R.; BOYCE, J.M.; FARR, B.M. SHEA guideline for preventing nosocomial transmission of multidrug-resistant strains of Sthaphylococcus aureus and Enterococcus. Infect. Control Hosp. Epidemiol., v.24, p.362-386, 2003.

QUAIN, R.D.; BARTON, T.D.; FISHMAN, N.O.; WEINER, M.G.; LAUTENBACH, E. Coadministration of oral levofloxacin with agents that impair its absorption: potential impact on emergence of resistance. Int. $J$. Antimicrob. Agents, v.26, n.4, p.327-330, 2005. 
RISSATO, M.A.R. Eventos adversos relacionados a medicamentos no contexto hospitalar: revisão da literatura. São Paulo, 2005. p.0142. [Dissertação de Mestrado. Faculdade de Saúde Pública. Universidade de São Paulo].

SANTELL, J.P.; COUSINS, D.D. Medication errors related to product names. Jt. Comm. J. Qual. Patient Saf., v.31, n.11, p.649-654, 2005.

SCHNEIDER, P.J. Opportunities for pharmacy. Am. J. Health Syst. Pharm., v.64, p.S10-S16, 2007.
TANG, F.I.; SHEU, S.S.; WEI, I.L.; CHEN, C. Nurse related the contributing factors involved in medication errors. $J$. Clin. Nurs., v.16, p.447-457, 2007.

WOLF, Z.R.; HICKS, R.; SEREMBUS, J.F. Characteristics of medication errors made by students during the administration phase: a descriptive study. J. Prof. Nurs., v.22, n.1, p.39-51, 2006.

WORLD HEALTH ORGANIZATION COLLABORATING CENTREFOR DRUG STATISTICS METHODOLOGY. Anatomical Therapeutic Chemical (ATC) index with Defined Daily Doses (DDDs). Disponível em: <http:// www.whocc.no/atcddd>. Acesso em: 10 mar. 2007.

Recebido para publicação em 29 de outubro de 2007 Aceito para publicação em 18 de dezembro de 2007 